\title{
The significance of the amoebocyte-producing organ in Biomphalaria glabrata
}

\author{
Samaly dos Santos Souza, Zilton Araújo Andrade/ ${ }^{+}$ \\ Laboratório de Patologia Experimental, Centro de Pesquisa Gonçalo Moniz-Fiocruz, \\ Rua Valdemar Falcão 121, 40295-001 Salvador, BA, Brasil
}

\begin{abstract}
In molluscs, internal defence against microorganisms is performed by a single cell type, i.e., the haemocyte or amoebocyte. The origin of these cells in Biomphalaria glabrata was initially thought to be localised within the vasculo-connective tissue. More recently, origin from a single organ, termed the amoebocyte-producing organ (APO), has been postulated based on the occurrence of hyperplasia and mitoses during Schistosoma mansoni infection. The present investigation represents a histological, immuno-histochemical and ultra-structural study of the B. glabrata APO, whereby histological identification was facilitated by means of collecting epithelial basophilic cells. These cells were comprised of single-cell layers that cover a portion of the stroma, which contains many small, round cells and haemolymph sinuses, as well as a small area of the pericardial surface of the reno-pericardial region. On occasion, this epithelial component vaguely resembled the vertebrate juxtaglomerular apparatus, which reinforces its presumed relationship to the kidney. Both in normal and infected molluscs, mitoses were only occasionally found. The present quantitative studies failed to demonstrate the presence of APO cellular hyperplasia, either in normal or schistosome-infected $\mathrm{B}$. glabrata. Conversely, several structural details from the APO region in $\mathrm{B}$. glabrata were found to be consistent with the hypothesis that the APO is a filtration organ, i.e., it is more closely related to the kidney rather than the bone marrow, as has been suggested in the literature.
\end{abstract}

Key words: haemocytes - Biomphalaria glabrata - Schistosoma mansoni - juxtaglomerular apparatus

The mollusc Biomphalaria glabrata is an important intermediate host for Schistosoma mansoni, which is the most important vector for human schistosomiasis in Brazil (Paraense 2001). The degree of resistance or susceptibility of the mollusc towards the parasite is variable and dependent on several factors, such as the defence cells contained within the mollusc. These defence cells belong to a single cell type, i.e., the amoebocyte or haemocyte. These cells circulate within the haemolymph and are present within the interstitial tissues of the mollusc and contribute to phagocytic and scavenging functions. Their site of origin has not yet been established with certainty, although initially, the data suggested a multicentric origin for amoebocytes (Wagge 1955). In keeping with previous embryologic findings in vertebrates, the amoebocytes were thought to emerge from the lining of the vascular spaces and cells of interstitial tissues within the tissues of the mollusc (Sminia 1972). Conversely, it has been reported more recently that during a schistosome infection, cellular hyperplasia and mitoses occur within a peculiar structure located at the reno-pericardial region, which has been termed the amoebocyte-producing organ (APO) (Lie \& Heyneman 1975, Lie et al. 1975). The present paper reports on the structure, nature and significance of the APO in normal and $S$. mansoni-infected B. glabrata molluscs.

+ Corresponding author: zilton@bahia.fiocruz.br Received: 23 August 2011

Accepted: 17 April 2012

\section{MATERIALS AND METHODS}

Samples of highly susceptible, laboratory-raised $B$. glabrata (Feira de Santana strain) (Andrade \& Sadigursky 1985), which were grown to $11-13 \mathrm{~mm}$ in diameter, were used for the experiments. Fifty snails were exposed to infection with approximately 20 recently shed $S$. mansoni miracidia, whereas 25 non-infected snails were maintained as controls. Groups of infected snails were submitted to dissection and fixation at six, 15 and 35 days following exposure to miracidial infection and the dissection and fixation process always included the non-infected control snails for comparison. The snails were anaesthetised with menthol prior to dissection. The procedure began by gently breaking the mollusc shell between two glass slides and removing the soft tissue with dissection forceps and then the haemolymph was collected using of a Pasteur pipette. The mollusc soft tissues were rinsed with buffered saline at $\mathrm{pH} 7.2$ and selected portions were fixed in $10 \%$ buffered formalin at $\mathrm{pH} 7.2$ and subsequently processed for paraffin embedding. After locating the heart, the reno-pericardial zone was dissected under a microscope. The $5-\mu \mathrm{m}$ thick paraffin sections from the renopericardial area were stained with haematoxylin-eosin.

Electron microscopy - For transmission electron microscopy, tiny portions from the reno-pericardial zone were immediately fixed by immersion into Karnovich solution for 3-4 $\mathrm{h}$ at room temperature (RT). The postfixation step was performed with a mixture of $2 \%$ osmium tetroxide and $0.4 \mathrm{M}$ cacodylate buffer at $\mathrm{pH} 7.4$ for $1 \mathrm{~h}$ at $4^{\circ} \mathrm{C}$. Subsequently, the samples were washed in CBSS solution, dehydrated in graded concentrations of acetone and embedded in Polybed 812. Selected ultra- 
thin sections were collected in copper grids, contrasted with uranyl acetate and lead citrate and examined under a Zeiss EM-9 electron microscope at an acceleration voltage of $50 \mathrm{kV}$.

Immunohistochemical procedures (actin) - The formalin-fixed, paraffin-embedded sections were placed on silanised slides. Antigen retrieval was performed using citrate buffer at $\mathrm{pH} 6.0$ and the blocking of endogenous peroxidase was performed with $0.3 \% \mathrm{H}_{2} \mathrm{O}_{2}$ for $30 \mathrm{~min}$ at RT. A 20 -min treatment at RT with $10 \%$ skim milk was used to eliminate non-specific ligations. After washing in phosphate buffered saline, the sections were incubated overnight with the diluted primary Dako (clone 1A 4) actin antibody at $4^{\circ} \mathrm{C}$. The slides were then incubated with the secondary antibody peroxidase-conjugated sheep anti-rat $\operatorname{IgG}$ (Dako envision system-labelled polymer) for $30 \mathrm{~min}$ at $37^{\circ} \mathrm{C}$ in a humidified chamber. The colour was developed with a DAB kit from DAKO and the sections were counterstained with haematoxylin.

Haemocyte counting - A 10- $\mu \mathrm{L}$ sample of lymph from each snail was collected and mixed with $90 \mathrm{~mL}$ neutral red. Ten millilitres of this solution was then placed on a Neubauer chamber and examined with a Leica Galen III optical microscope to count the number and types of cells at $40 \mathrm{X}$ magnification. The counting procedure was performed independently by two observers.

\section{RESULTS}

APO morphology - The optical microscopy studies indicated that the structure considered to represent the APO demonstrated the presence of epithelial cells, which formed either single cell-thick rows or clusters exhibiting a dark colour when stained with haematoxylin. These cell clusters were delimited by thin tracts of connective tissue covering the superior and lateral portions of the pericardial sac. On average, the tissue thickness was determined according to the presence of five-seven cell layers. Additionally, lymph sinuses were observed between the cells of the APO, which likely enable the passage of lymph (Fig. 1B).

In the tissues of infected animals, some sporocysts and cercariae were observed at various developmental stages and these were observed both with and without evident cellular responses surrounding them. Such findings were also observed within the APO and, in these cases, it was possible to identify parasitic structures or pigmented remains between the cells. The APO cells exhibited no apparent cellular response, as neither enlargement nor hyperplasia was observed in the band occupied by this structure. Furthermore, even after an exhaustive search using the 100X objective, few mitotic figures were detected in the cells of the APO region (Fig. 1C, D).

Through the use of Giemsa and Masson trichrome staining, the chromosomes of the APO cell nuclei were shown to be generally very large and composed of thick strands of chromatin, which were strongly stained in blue, in contrast to the pale blue-stained cytoplasm. With Masson's trichrome stain, both the APO cells covering the pericardial ribbon and the epithelial tissue border- ing the renal portion were stained cherry red, whereas the walls of connective tissue delimiting these cells were stained blue. These findings were observed in both the APOs of normal and infected snails.

Immunohistochemistry of the APO - An anti-actin antibody typically used to stain smooth muscle tissue was used to stain the APO and this staining was positive for the APO region, which suggests a possible contractile function for this structure (Fig. 1E).

Haemolymph - The two types of haemocytes, hyalinocytes and granulocytes, were identified in the lymph collected from normal and infected snails. The hyalinocytes appeared to be immature, spherical and small, whereas the granulocytes exhibited eccentric nuclei and an expanded cytoplasm with filopodia (Fig. 1F).

Statistics - When comparing the two types of haemocytes within the same group, namely haemocytes of the normal group (hyalinocytes $x$ granulocytes) using the unpaired $\mathrm{T}$ test, granulocytes were present in significantly greater numbers in all samples as compared to hyalinocytes $(p<0.05)$. Similar findings were observed for the types of haemocytes found in infected snails after different time periods of exposure to the miracidia of $S$. mansoni (Fig. 2A). The numbers of circulating haemocytes between the infected and normal snails at six, 15 and 30 days post-infection were not significantly different, as these numbers averaged $1.40 \times 10^{4}, 2.50 \times 10^{4}$, $1.80 \times 10^{4}$ and $3.00 \times 10^{4}$, respectively. However, according to the Newman-Keuls post-hoc test, the numbers of haemocytes from the uninfected snail group differed significantly from those in the infected group at 30 days post-infection $(p<0.01)$ and the numbers of haemocytes in the infected group of snails at 15 days post-infection differed significantly from those in the infected group of snails at 30 days post-infection ( $\mathrm{p}<0.05$ ) (Fig. $2 \mathrm{~B}$ ).

Electron microscopy - Electron microscopy revealed that the APO cells exhibited an apical zone with microvilli on the free surface, which is a typical characteristic of epithelial tissue. In the outer membrane, the presence of numerous interdigitations and desmosomes delimited the border and anchored each cell to the other. Also, eccentric nuclei with condensed chromatin were observed and the cytoplasm was shown to contain mitochondria of different sizes and numerous electrondense granules (Fig. 3A). The dense cytoplasm also contained smooth endoplasmic reticulum as well as rough endoplasmic reticulum, the Golgi complex, autophagic vacuoles, a few lysosomes, vacuoles of fat and myelin figures (Fig. 3B). These cells were large, individualised and were observed resting on a thick basement membrane. Underlying this structure, we observed connective tissue containing glycogen rosettes and tonofibrils, as well as structures that were identified as microtubules (Fig. 3C). Some amoebocytes were also observed within the sub-epithelial tissue of the APO. In this region, the amoeboblasts exhibited clearly visible filopodia, a delicate cytoplasm and an eccentric nucleus, in which it was possible to observe the presence of a membrane, the for- 

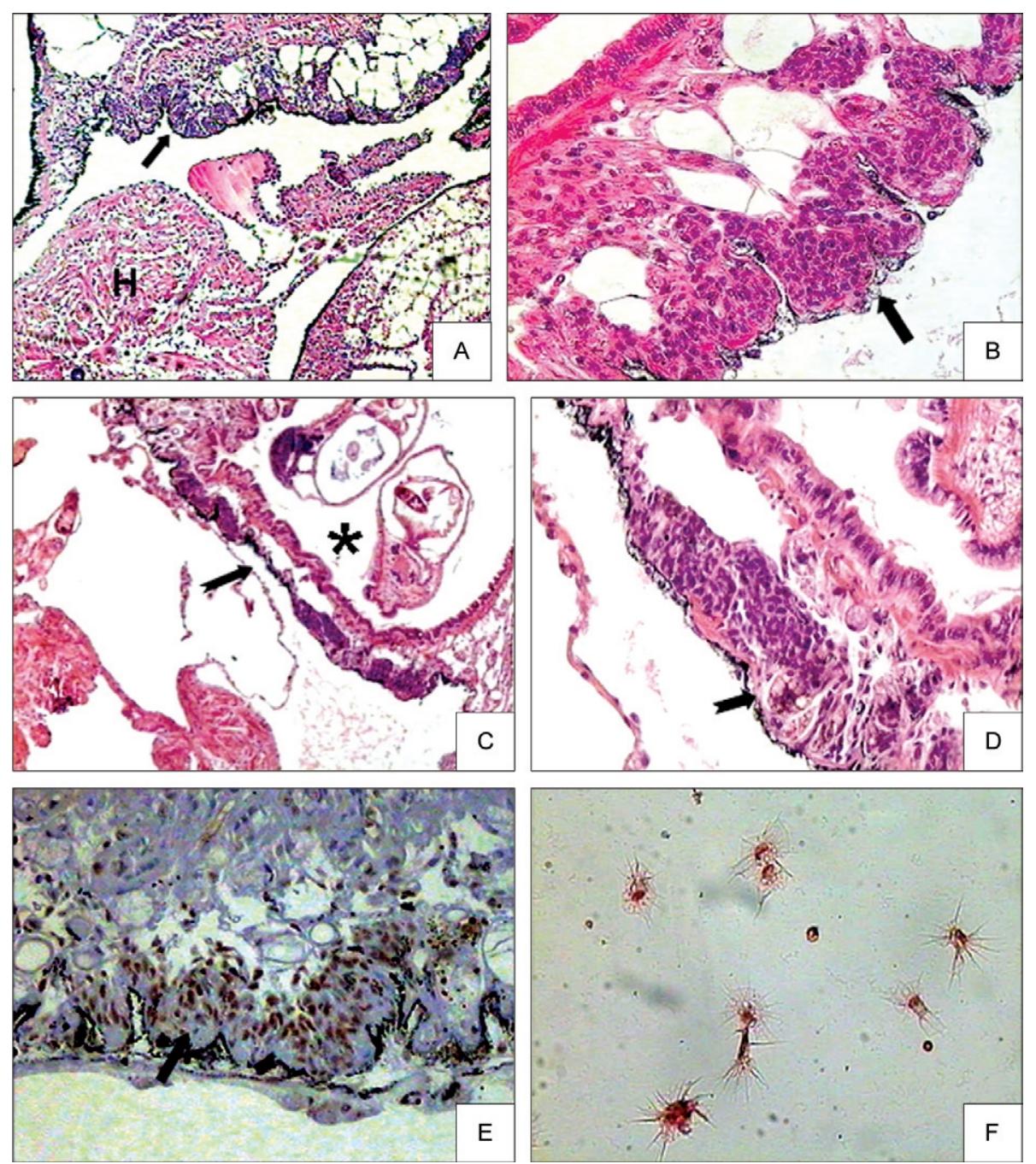

Fig. 1A: amoebocyte producing organ (APO) from normal Biomphalaria glabrata. Reno-pericardial zone (arrow) situated between the saccular zone of the kidney and the heart (H) (200X); B: APO, epithelial cells delimited by thin fibrous septa and vascular spaces (arrow) (400X); C: APO from Schistosoma mansoni-infected B. glabrata. No evidences of cellular proliferation (arrow), presence of sporocyst near the APO (mark) (200X); D: APO - presence of parasite (arrow), but no signs of cellular hyperplasia (200X); E: APO cells stained dark brown positive for $\alpha$-actin (arrow) (400X); F: haemocytes from circulating lymph. Hyalinocytes are spherical and tiny while granulocytes are spherical and small with cytoplasmic prolongations (neutral-red) (200X).

mation of myelin figures with adjacent granular material (most likely a ribosome) and a large autophagic vacuole. Additionally, haemolymphatic spaces and the presence of numerous electron-dense bodies were observed (Fig. 3D). The structures found in the APO cells of infected snails were also observed in the APO cells of the uninfected controls. However, in the infected animals, the presence of numerous dense bodies with mitochondrial profiles, a granular matrix and elongated electron-lucent structures were more frequently observed (Fig. 3E).

\section{DISCUSSION}

Our present findings demonstrated no evidence to support the assertion that haemocytes in B. glabrata have a common origin from a central body, i.e., the APO, which has been proposed to be a type of bone marrow that produces the circulating cells responsible for defence in $B$. glabrata. Current research suggests that haemocytes can be formed at different locations in Pulmonata, such as within the connective tissue and vascular system of snails (Lymnaea stagnalis) (Sminia 1974, Sminia \& van der Knaap 1983) or, alternatively, in an organ located in a region anatomically close to the kidney (Bulinus sp. Kinoti 1971, Marisa cornuarietis Yousif et al. 1980). In addition, the APO has been shown to be located between the pericardium and the posterior epithelium of the mantle cavity in B. glabrata (Sullivan et al. 1984, 2004, Sullivan 1990) and Lymnaea catascopium (Loker 1979).

Moreover, studies by Pan $(1958,1963,1965)$ described this APO structure as comparable to a lymphoid tissue and the ultrastructure of the APO was first described by Jeong et al. (1983) as being similar to the bone 
A

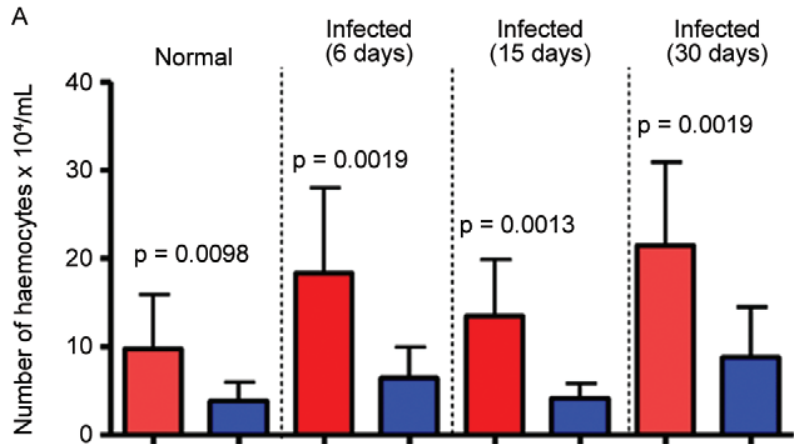

Granulocytes

Hyalinocytes

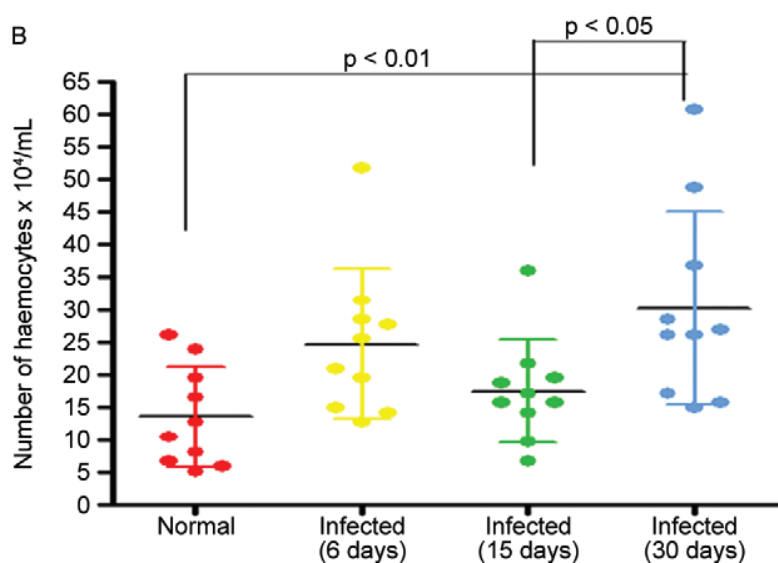

Fig. 2A: haemocytes (granulocytes and hyalinocytes) taken from fresh and normal haemolymph from Schistosoma mansoni infected Biomphalaria glabrata at different periods of infection (NewmanKeuls, 95\% confidence interval); B: haemocytes counts (average) made on fresh haemolymph taken from $S$. mansoni-infected $B$. glabrata at different periods of infection and in controls (NewmanKeuls, $95 \%$ confidence interval).

marrow of vertebrates. These studies preceded those of Sullivan et al. $(1984,2004)$ and Sullivan (1990) and observed hyperplasia and mitosis in cells of the APO during S. mansoni infection. Another piece of evidence suggesting that the APO could be a haemocyte-producing organ came from studies performing APO transplants from an animal resistant to $S$. mansoni to a susceptible animal, whereby the susceptible animal subsequently became resistant (Sullivan \& Spence 1994). However, there are several limitations to this type of transplant, as nearby structures in addition to the APO may also be transplanted (Barbosa et al. 2006).

Furthermore, Souza and Andrade (2006) observed that the APO in B. glabrata, aside from being a frail microscopic structure, exhibited a structure that was impossible to dissect and isolate when viewed without microscopic magnification. Moreover, the APO demonstrated a multifocal distribution that occupied a very narrow band, which greatly hindered the identification of the sections that were taken and often required the use of a series of sections. In the current study, large numbers of haemocytes were not observed in either the normal animals or S. mansoni-infected animals and this was true both within and in the immediate vicinity of the APO and even in animals where parasitic structures were found inside of the APO. However, collections of amoebocytes were found in the linings of the vascular spaces of other organs and these cells exhibited signs of multiplication, such as morphologic evidence of progressive detachment from the endothelial lining into the interior of the vascular spaces. Isolated collections of haemocytes in the saccular region of the kidney and the columellar region of the mantle were also found, which led us to conclude that haemocytes were produced in various regions of $B$. glabrata instead of in a single body.

Several analyses were also performed for the detection of mitotic cells in this organ and the results indicated that cells even from $B$. glabrata infected with $S$. mansoni were not undergoing proliferation. The numbers of circulating haemocytes in B. glabrata were similar between normal and infected snails. In addition, it would be expected that cells originating within a central organ would flow with the haemolymph to concentrate themselves at locations of infection. In this way, we had expected to observe a higher concentration of haemocytes in the haemolymph of infected animals, which would have represented a kind of "leukocytosis". However, the data collected thus far have not supported this hypothesis. In addition, other researchers have failed to demonstrate a consistent finding between the tissue response and its effect on cells from the haemolymph (Reis et al. 1995, Borges 2005). In addition, as first noted by Jeong et al. (1983), our ultrastructural findings demonstrated that the APO is a complex structure that is composed of juxtaposed cells with epithelial characteristics, such as microvilli, on its free surface. These cells are rich in organelles, which suggest not only an important metabolic function for this organ, but functions related to secretion and filtration as well. However, these cells did not provide evidence of their differentiation into free cells (haemocytes). Haemocytes were observed in the connective tissue just below the lower membrane of the APO, although the transitional forms of cells within this tissue were not examined. We also performed an ultrastructural study of other regions of the snail, such as the tentacle and columellar muscle of the mantle. In these structures, the cells demonstrated characteristics that were similar to those observed in the APO, such as an epithelial tissue with identical organelles, although these cells also exhibited an organisational arrangement with some peculiarities.

Thus, our findings do not support the hypothesis that the APO is an APO organ. In L. stagnalis (Sminia 1972, 1974), the APO was not highlighted as an APO, as these authors agreed that amoebocytes could be generated in the lymph and tissues. In Lymnaea truncatula (Rondelaud \& Barthe 1982, Monteil \& Matricon-Gondran 1990), it was shown that the APO exhibited the typical structure of a filtering organ, such as the kidney, but had no structural similarity to lymphoid tissue. Due 

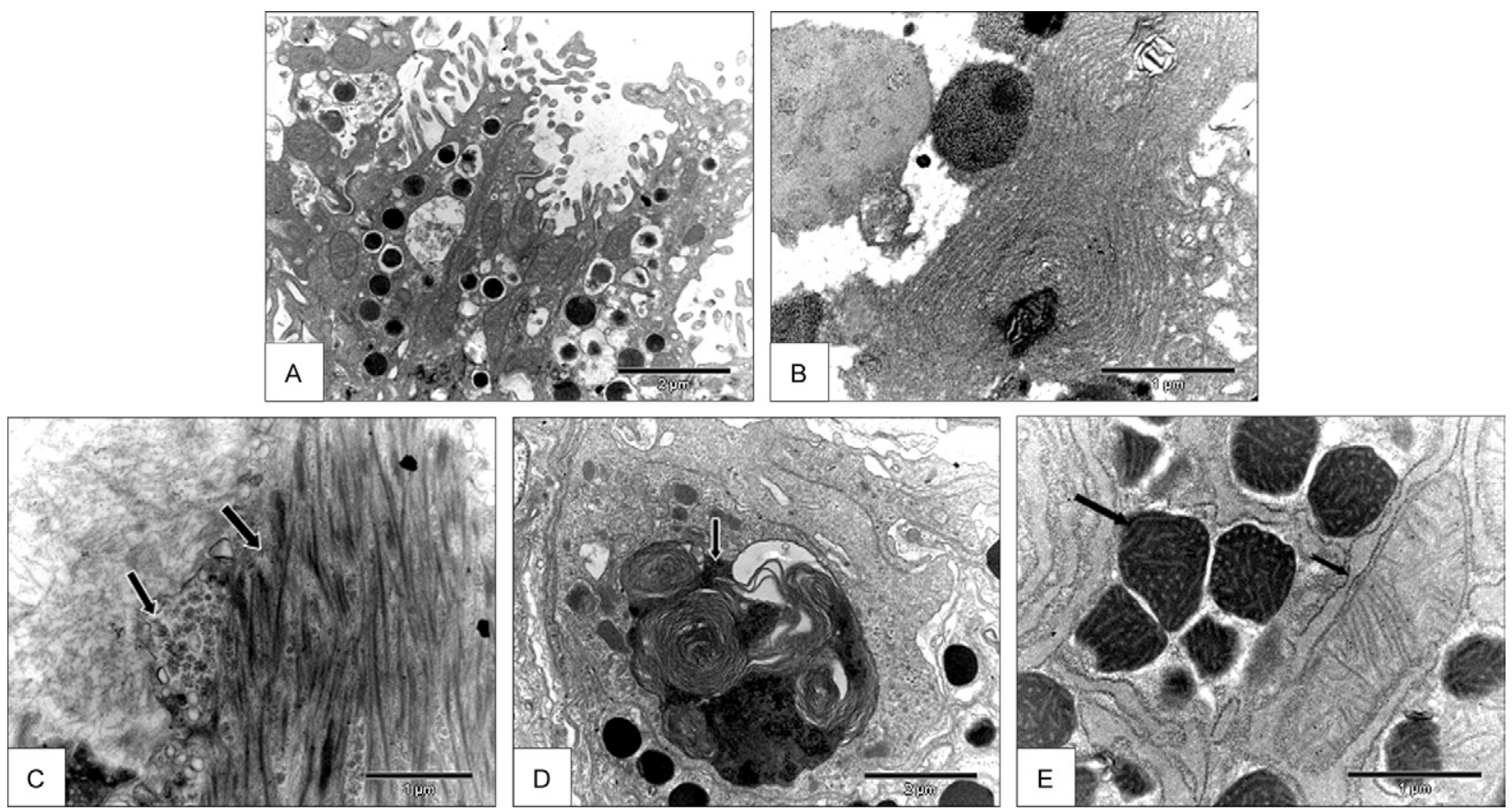

Fig. 3A: epithelial cells presenting microvilli at the free surfaces and desmosomes in between cells; B: rough endoplasmic reticulum, Golgi apparatus, myelin figures and autophagic vacuole can be seen; C: glycogen granules (arrow), actin filaments (arrow) and microtubule formation can be seen at the amoebocyte producing organ (APO); D: autophagic vacuole; E: presence of dense bodies, rough endoplasmic reticulum (arrows) and mitochondria (arrows) were more frequently seen in APO cells from infected snails.

to the APO location within the reno-pericardial region, the significant absence of mitoses and the presence of lipid droplets, autophagic vacuoles, haemolymphatic vessels and numerous granules led previous authors to describe the APO as a structure related to the secretion or excretion of Pulmonata. Furthermore, when the ultrastructure of the small area that forms the APO was analysed histologically, the cellular arrangement was notable, as the APO was formed by juxtaposed basophilic cells that were arranged in rows and exhibited epithelial cell characteristics, which were not observed in the other organs of the mollusc.

These findings for the cells of the APO are similar to those of cells in the juxtaglomerular apparatus present in the mammalian kidney, as both demonstrate spherical nuclei and a cytoplasm containing granules, abundant rough endoplasmic reticulum, secretory granules, a very well developed Golgi apparatus and many dense corpuscles. Moreover, these cells play an important role in the control of ionic balance.

Our findings favour a multicentric origin for the haemocyte in B. glabrata. These haemocytes could be formed in any organ and may originate from cells lining the venous sinuses or cells situated within loose connective tissue. Moreover, the basophilic epithelial ribbon that enables the identification of the APO area could potentially represent a structure related to the juxtaglomerular apparatus.

\section{ACKNOWLEDGEMENTS}

To Adriana Manfredi, for her invaluable help during the studies with the electron microscope.

\section{REFERENCES}

Andrade ZA, Sadigursky M 1985. Um estudo comparativo das cepas Feira de Santana (Bahia) e Porto Rico do Schistosoma mansoni na infecção experimental do camundongo. Mem Inst Oswaldo Cruz 80: 37-40.

Barbosa L, Caldeira RL, Carvalho OS, Vidigal THDA, Jannotti-Passos LK, Coelho PMZ 2006. Resistance to Schistosoma mansoni by transplantation of APO Biomphalaria tenagophila. Parasite Immunology 28: 209-212.

Borges CMC 2005. Hemócitos de Biomphalaria glabrata (Say, 1818) na interação com o Schistosoma mansoni Sambom, 1907, PhD Thesis, Universidade Federal da Bahia, Salvador, 221 pp.

Jeong KH, Lie KJ, Heyneman D 1983. The ultrastructure of the amoebocyte-producing organ in Biomphalaria glabrata. Dev Comp Immunol 7: 217-228.

Kinoti GK 1971. Observations on the infection of bulinid snails with Schistosoma mattheei. Parasitology 62: 161-170.

Lie KJ, Heyneman D 1975. Studies on resistance in snails: a specific tissue reaction to Echinostoma lindoense in Biomphalaria glabrata. Int J Parasitol 15: 621-626.

Lie KJ, Heyneman D, Yau D 1975. The origin of amoebocytes in Biomphalaria glabrata. J Parasitol 61: 574-576.

Loker ES 1979. Pathology and host responses induced by Schistosomatium douthitti in the freshwater snail Lymnae catascopium. J Invertebr Pathol 33: 265.

Monteil JF, Matricon-Gondran 1990. Haemocyte production in trematode-infected Lymnaea truncatula. Parasitol Res 77: 491-497.

Pan CT 1958. The general histology and topographic microanatomy of Australorbis glabrata. Bull Mus Comp Zool 119: 237-299. 
Pan CT 1963. Generalized and focal tissue responses in the snail, Australorbis glabratus, infected with Schistosoma mansoni. Ann NY Acad Sci 113: 475-485.

Pan CT 1965. Studies on the host-parasite relationship between Schistosoma mansoni and the snail Australorbis glabrata. Am J Trop Med Hyg 14: 931-975.

Paraense WL 2001. The schistosome vectors in the Americas. Mem Inst Oswaldo Cruz 96 (Suppl.): 7-16.

Reis SMPM, Magalhães LA, Carvalho JF 1995. Ação da inoculação de hemolinfa no mecanismo de defesa de Biomphalaria tenagophila. Rev Saude Publica 29: 259-264.

Rondelaud D, Barthe D 1982. Relationship of the amoebocyte-producing organ with the generalized amoebocyte reaction in Lymnaea truncatula Muller infected by Fasciola hepatica. L J Parasitol 68: 967-969.

Sminia T 1972. Structure and function of blood and connective tissue cells of the fresh water pulmonate Lymnaea stagnalis studied by electron microscopy and enzyme histochemistry. Z Zellforsch 130: 497-526.

Sminia T 1974. Haematopoiesis in the freshwater snail Lymnaea stagnalis studied by electron microscopy and autoradiography. Cell Tiss Res 150: 443-454.
Sminia T, van der Knaap WPW 1983. Blood cell types and blood cell formation in gastropod mollusks. Dev Comp Immunol 7: 665-668.

Souza S dos S, Andrade ZA 2006. On the origin of the Biomphalaria glabrata haemocytes. Mem Inst Oswaldo Cruz 101 (Suppl. I): 213-216.

Sullivan JT 1990. Long-term survival of heterotypic allografts of the amoebocyte-producing organ In Biomphalaria glabrata (Mollusca: Pulmonata). Trans Am Microsc Soc 109: 52-60.

Sullivan JT, Cheng TC, Howland KH 1984. Mitotic responses of the anterior pericardial wall of Biomphalaria glabrata (Molusca) subjected to challeng. J Invertebr Patho 44: 114-116.

Sullivan JT, Pikios SS, Alonzo AQ 2004. Mitotic responses to extracts of miracidia and cercariae of Schistosoma mansoni in the amoebocyte-producing organ of the snail intermediate host $B i$ omphalaria glabrata. J Parasitol 90: 92-96.

Sullivan JT, Spence JV 1994. Transfer of resistance to Schistosoma mansoni in Biomphalaria glabrata by allografts of amoebocyteproducing organ. $J$ Parasitol 80: 449-453.

Wagge LE 1955. Amoebocytes. Int Rev Cytol 4: 31-78.

Yousif f, Blahser S, Lammler 1980. The cellular responses in Marisa cornuarietis experimentally infected with Angiostrongylus cantonensis. Parasitol Res 62: 179-190. 